\title{
Simulación Clínica en Enfermería. Un enfoque para la práctica pedagógica del acto de cuidar
}

\author{
Jorge Omar Trisca *, Rosana Elisabet Firpo **, Emiliano Argüello ***
}

\section{Resumen}

La Simulación Clínica como estrategia educativa constituye una enorme oportunidad para revisar las prácticas pedagógicas de las disciplinas del área de la salud en general pero de la Enfermería en particular.

En este sentido, el trabajo en conjunto con las Ciencias de la Educación ofrece un campo fértil para proponer enfoques que contribuyan a reformular los roles de docentes y estudiantes. Si bien hay un acuerdo en identificar el aprendizaje significativo como la principal orientación pedagógica, la mención de autores como Kolb y Schön sólo ofrecen un marco general en lo que respecta al proceso enseñanza-aprendizajeevaluación del cuidado enfermero.

Desde este artículo se propone el enfoque del Aprendizaje Pleno de David Perkins que, en la misma línea del aprendizaje experiencial, contribuye específicamente a la Enfermería al proponer jugar el juego completo, lo que habilita la inclusión de un modelo disciplinar en la resolución de casos clínicos como puntapié para las actividades de simulación.

Cabe aclarar que la propuesta surge fundamentalmente, a partir de los resultados obtenidos en un proyecto de investigación que se desarrolló en el año 2018 denominado "La utilización de simuladores en la enseñanza en Enfermería. Salir de la racionalidad instrumental a través de nuevas prácticas pedagógicas" y se acompañó de la realización de Talleres de cuidados en lo que a la docencia respecta. Además, se presentó y publicó la experiencia en varios eventos académico científicos de nivel provincial (Entre Ríos), nacional e internacional lo cual demuestra el estrecho enlace entre las actividades que corresponden al nivel superior: la docencia, la investigación y la extensión.

En el caso específico de este artículo se presenta una introducción desde las Ciencias de la Educación, se detallan los principios de Perkins en relación a las etapas de la Simulación Clínica y se comparte el testimonio de un Coordinador de un Centro de Simulación con el propósito de orientar un recorrido heurístico en la docencia en Enfermería.

Se sugiere que esta unión entre salud y educación se acompañe de investigación en la que participen las carreras tanto de pregrado como de posgrado y los Centros de Simulación a fin de validar resultados y verificar el impacto en el proceso enseñanzaaprendizaje-evaluación del cuidado enfermero.

Palabras clave: $\quad$ Simulación clínica - enfoque pedagógico - Aprendizaje Pleno - Cuidado enfermero 


\title{
Clinical Simulation in Nursing. An approach to the pedagogical practice of the act of caring
}

\author{
Jorge Omar Trisca *, Rosana Elisabet Firpo **, Emiliano Argüello ***
}

\begin{abstract}
The Clinical Simulation as an educational strategy constitutes an enormous opportunity to review the pedagogical practices of the disciplines of the health area in general but of Nursing in particular.

In this sense, working together with Education Sciences offers a fertile field to propose approaches that contribute to reformulating the roles of teachers and students. Although there is an agreement in identifying meaningful learning as the main pedagogical orientation, the mention of authors such as Kolb and Schön only offers a general framework regarding the teaching-learning-evaluation process of nursing care.

This article proposes the approach of Full Learning by David Perkins that, in the same line of experiential learning, specifically contributes to Nursing by proposing to play the full game, which enables the inclusion of a disciplinary model in the resolution of clinical cases as a kickstart for simulation activities.

It should be clarified that the proposal arises fundamentally, from the results obtained in a research project that was developed in 2018 called "The use of simulators in nursing teaching. Get out of instrumental rationality through new pedagogical practices "and it was accompanied by the realization of care workshops with regard to teaching. In addition, the experience in various academic-scientific events at the provincial (Entre Ríos), national and international level was presented and published, which shows the close link between the activities that correspond to the higher level: teaching, research and extension.

In the specific case of this article, an introduction from the Sciences of Education is presented, the Perkins principles in relation to the stages of Clinical Simulation are detailed and the testimony of a Coordinator of a Simulation Center is shared with the purpose of guide a heuristic journey in nursing teaching.

It is suggested that this union between health and education be accompanied by research involving both undergraduate and graduate careers and Simulation Centers in order to validate results and verify the impact on the teaching-learning-evaluation process of nursing care.
\end{abstract}

Keywords: Clinical simulation - pedagogical approach - Full Learning - Nursing care 


\section{Fundamento pedagógico para la estrategia educativa de la Simulación Clínica}

Todo proceso educativo necesita de sólidos apuntalamientos provenientes de la pedagogía y esto es imprescindible para que este proceso se constituya en una empresa exitosa.

Cuando de la simulación clínica se trata, no solo es imprescindible poseer los recursos didácticos adecuados, sino que también la práctica educativa debe transitar por procesos que permitan asegurar dentro de lo previsible, que los alumnos están adquiriendo un aprendizaje significativo.

Los fundamentos pedagógicos naturalmente deben enfocarse en que los estudiantes sean capaces de comprender las implicancias y las derivaciones que tienen su accionar en la salud de las personas $y$, por tanto, la praxis educativa debe dotar al estudiante no solo de pericia en el manejo de las diferentes técnicas a su alcance sino, y lo más importante, ser autónomos y pensadores profundos para tomar las decisiones correctas en los momentos críticos.

En primer lugar, las competencias se desarrollan cuando se coloca a los estudiantes en situaciones problemáticas que exijan la toma de decisiones (Perrenoud, 2007) una manera de lograrlo puede ser mediante la metodología de proyectos o a través de la incorporación de actividades no convencionales a las clases (Stiefel, 2008) pero lo fundamental es que las formas de enseñanza puedan dar respuesta a situaciones, problemas o conflictos surgidos de la vida real (Zabala y Arnau, 2010).

Concretamente, el objetivo de este trabajo es analizar y proponer un cambio educativo mediante la implementación en la vida áulica y en el laboratorio de simulación, las estrategias que contribuyan al desarrollo de competencias.

En este caso en particular, resulta muy atractivo incorporar los principios de Perkins en lo relativo a cómo conviene presentar los procedimientos a aprender de manera que sean incorporados por parte de los estudiantes de una manera provechosa.

Estos principios del aprendizaje reaccionan fundamentalmente contra la creencia muy difundida que los contenidos deben impartirse de modo fragmentado (propio del paradigma conductista) para una mejor asimilación o, por otra parte, enfatizar primero los aspectos teóricos para luego llevarlos a la práctica en un centro de simulación donde se espera que los estudiantes apliquen lo estudiado previamente.

Pero si nos enfocamos en un proyecto educativo contemporáneo, donde interesa el desarrollo integral de los estudiantes, entonces la perspectiva de la formación debe cambiar radicalmente. En otras palabras, desarrollo significa que los alumnos adquieran principios que les permita acceder a una vida trascendental, es decir más allá de su propia realidad. Además, que obtengan la preparación necesaria para poder desenvolverse con éxito y mejorar la sociedad en que viven. En este contexto, el término íntegro connota el sentido semántico de cosa única, indisoluble, una unidad indivisible (Darós, 1994), no como partes de un todo. En los años noventa, la Organización de las Naciones Unidas para 
la Educación, la Ciencia y la Cultura (UNESCO) formó una comisión internacional que debía estudiar y señalar los patrones en los cuales se basaría la educación para el Siglo XXI. La comisión concluyó que la educación del siglo venidero tendría que cimentarse en cuatro pilares: aprender a conocer, aprender a hacer, aprender a vivir juntos, aprender a ser (Delors, 1997). De allí que responsabilidad de las universidades para este nuevo siglo incluyera también, formar integralmente a cada educando. En síntesis, pensar en una educación integral implica atender el desarrollo de todas las potencialidades que cada ser humano posee y la educación por competencias, justamente, se enfoca en que cada dimensión de la persona alcance su máxima performance.

Muchas universidades tienen dificultades en áreas como cobertura e inclusión, pero también en aspectos relacionados con pertinencia, eficacia, flexibilidad e innovación. En este marco surgen voces como la de Schmidt (2010), docente de la Universidad de las Naciones Unidas, quien plantea que los actuales modelos de la educación (superior) están en crisis, a causa de problemas como: el desfase entre las habilidades enseñadas y las requeridas en el mundo técnico-profesional; un alza desmesurada del precio de las matrículas; una formación de corto alcance que no prepara adecuadamente para los desafíos del mañana; o la adopción de planes de formación rígidos, fragmentados y expuestos a quedar obsoletos tras tres o cuatro años de estudio (Cobo \& Moravec, 2011).

Por ejemplo, en el área de las ciencias fácticas como la química, existen muchos conceptos que son abstractos y de difícil comprensión para los estudiantes porque generalmente carecen de organizadores previos. Por lo tanto, adquieren de errores conceptuales que son complicados de erradicar o bien se inclinan por aprendizajes fragmentados, sin sentido y basado en la memoria exclusivamente. Sin embargo, si los métodos de enseñanza son adaptados a los intereses y estímulos de los estudiantes hay buenas posibilidades que el aprendizaje mejore.

Según Coll (1997) las investigaciones llevadas a cabo en las dos últimas décadas del siglo pasado coinciden en señalar que la actividad cooperativa en las escuelas inciden de forma decisiva en el proceso de socialización en general, igualmente para la adquisición de competencias y de destrezas y ayuda a un mejor manejo del control de los impulsos agresivos, facilitando la adaptación a las normas establecidas, beneficiando incluso el rendimiento escolar, además de otros aspectos cualitativos relacionados con el autoconocimiento. Además, los alumnos integrantes de grupos bien estructurados consideran que el aprendizaje es más divertido (Woolfolk, 2010). Por otra parte, Ferreiro (2007) señala que esta propuesta es una de las más innovadoras en materia de educación que ha producido el constructivismo social. Dicho en otras palabras, hay trabajo cooperativo cuando los alumnos de un grupo escolar concilian ideas, aunque tengan puntos de vista diferentes o divergentes y este proceso constructivo de conocimiento permite que cada individuo aprenda más de lo que aprendería individualmente, justamente por la interacción de los integrantes del equipo (Duart y Sangrà, 2005).

Por otro lado, varias investigaciones han demostrado que los grupos cooperativos favorecen más el aprendizaje que el trabajo individual (Medina Gonzáles, 2017; Johnson, 
Johnson \& Holubec, 1999). De hecho, la interacción de los integrantes es un proceso clave en la construcción del conocimiento. Pero para que esto ocurra, es necesario el empleo de estrategias didácticas adecuadas para lograr como producto final y habitual, el aprendizaje colaborativo (Revelo-Sánchez, Collazos-Ordóñez, y Jiménez-Toledo, 2018). En efecto, aprender colaborativamente, significa que todos los participantes actúan de manera solidaria y aportan para el crecimiento grupal. Cada integrante del grupo colaborativo asume una responsabilidad individual en cuanto a su propio aprendizaje y el de los demás integrantes de modo que puede percibirse una gran motivación intrínseca (Trisca, 2014). Por lo tanto, aprender a trabajar colaborativamente es una competencia indispensable para preparar profesionales que puedan integrarse fácilmente a la dinámica de las sociedades modernas.

La simulación puede definirse como "una estrategia educativa en la que unos conjuntos particulares de condiciones son creadas o replicadas para asemejar situaciones auténticas que son posibles en la vida real" (INACSL, 2016: 44). A partir de la incorporación de la Simulación Clínica, el proceso enseñanza/aprendizaje/evaluación del cuidado enfermero se presentan tensiones, desafíos y oportunidades para las prácticas pedagógicas que requieren repensarse desde nuevos enfoques como el de Perkins.

Cuando se presentó el informe final del proyecto de investigación "La utilización de simuladores en la enseñanza en Enfermería. Salir de la racionalidad instrumental a través de nuevas prácticas pedagógicas" se subrayó el enlace entre la docencia y la investigación a partir del cual se propone una innovación pedagógica.

La formulación del problema: “¿Cómo impacta la inclusión de la simulación como estrategia de enseñanza en los roles que asumen docentes y estudiantes en los espacios de Práctica Profesionalizante de la Tecnicatura en Enfermería?" surgió a partir de observar la vigencia del modelo biomédico en el ámbito educativo. Como objetivo general se planteó: analizar la inclusión de la simulación como estrategia de enseñanza y su impacto en la redefinición de los roles de docentes y estudiantes y el universo lo constituían los/as docentes y estudiantes en los espacios curriculares de Prácticas Profesionalizantes I, II y III realizando un muestreo aleatorio en el caso de los/as estudiantes.

Si bien la metodología propuesta en un inicio fue realizar encuestas y entrevistas, ya en la prueba piloto se observó una baja tasa de respuestas atribuida principalmente a una cultura institucional ajena a la investigación ya que en catorce años de vigencia de la Tecnicatura era la primera vez que había un proyecto. Por lo tanto se optó por generar otras fuentes de datos como el análisis de las planificaciones de cátedra, acceder a muestras de oportunidad y generar espacios de investigación/acción como un Taller de cuidados en Gestosis ${ }^{1}$.

\footnotetext{
${ }^{1}$ De hecho, también fue la única experiencia de investigación al día de hoy ya que los/as responsables del Área de Investigación de la provincia, dependientes del Consejo General de Educación optaron por asignar todas las horas a los Profesorados en los años subsiguientes. En la experiencia realizada para el Taller de cuidados en Gestosis, el escenario se armó en el Hospital local y los/as estudiantes resaltaron que "es la primera vez que vamos a un hospital sin la presión de atender un paciente". El diseño del Taller permitió
} 
De esta forma, una de las principales conclusiones del proyecto fue la preocupación por la forma en que la Simulación Clínica se incorpora al pregrado en Enfermería y la oportunidad de aplicar un enfoque pedagógico a través del Aprendizaje Pleno.

\section{Los siete principios del Aprendizaje Pleno aplicados a la Simulación Clínica}

Una de las primeras cuestiones que Perkins (2016) se ocupa cuidadosamente de señalar antes de describir los siete principios que constituyen la base de su teoría es la relacionada con el abordaje de la complejidad. En ese sentido puede hacerse mención a que "en la práctica de Enfermería se observa que el cuidado se identifica en el hacer monótono, lineal y sin sentido dentro de la racionalidad técnica y el enfoque biomédico (...) situación que propicia la negación de la Enfermería como ciencia y como arte" (BáezHernández y colaboradores, 2009: 129).

Por el contrario, desde este trabajo se postula al objeto de estudio y trabajo de la disciplina como un objeto complejo y esta dimensión debería ser recuperada en las prácticas pedagógicas. Por lo expuesto, y asociado a las consideraciones bioéticas de la utilización de simuladores, Peña Jiménez y Amador Aguilar (2015: 158) resaltan la necesidad de un adecuado desarrollo didáctico ante el riesgo de cosificar el cuidado e instan a que los escenarios que se diseñen incorporen la complejidad que se deriva del acto de cuidar a una persona.

En consonancia con lo anterior, y lejos de considerar que la Enfermería resulta de la enseñanza-aprendizaje de técnicas y procedimientos y memorización de patologías, es que se invoca a la responsabilidad de los/as educadores/as de Enfermería en relación a la planificación de escenarios que tiendan a promover el pensamiento crítico ya que "los graduados en Enfermería deben estar preparados para ejercer (...) en un entorno altamente complejo, diverso y de constante cambio" (Sharma, 2017: 1).

De lo anterior se deriva que no es lo mismo planificar la colocación de una vía endovenosa sin relatar quién es la persona que necesita esa vía y en qué circunstancias: ¿un anciano que concurre a la guardia deshidratado? ¿O un joven que sufrió un accidente de moto y necesita ser transfundido? Si la idea es, tal como lo plantean Bland y colaboradores (2011) una propuesta que involucre tanto el aprendizaje activo como el pensamiento creativo y tienda a la resolución de problemas de alto nivel, necesariamente se debe partir de un caso clínico a partir del cual trabajar con un modelo disciplinar. Y eso es lo que se propone desde el Aprendizaje Pleno cuyos principios se exponen a continuación:

Primer principio: Jugar el juego completo: Perkins le atribuye a este punto que el avance en el aprendizaje sea significativo y motivador y un enlace con la comprensión de todo el proceso. Además, el autor aclara que "el desafío de jugar el juego completo no 
consiste en encontrar la versión oficial correcta y canónica sino en poner en práctica alguna versión razonable" (2016: 55 ) con lo cual abre la puerta para el diseño de casos clínicos de diferente grado de complejidad que permitan valorar los diferentes tipos de competencias: cognoscitivas, procedimentales y relacionales. No sólo eso; a partir de presentar un caso, éste se puede resolver mediante cualquier modelo disciplinar aunque desde este trabajo se oriente la incorporación del Modelo de Roper, Logan y Tierney (1993).

El hecho de diseñar un caso clínico como punto de partida para las actividades de Simulación Clínica tiene que ver con abordar desde la complejidad y no desde la especificidad de realizar una técnica o procedimiento aislado y por otra parte con recuperar el concepto de transferencia según el cual "el aprendizaje inicial debe incluir una cuota de la conexión que esperamos que los alumnos hagan más adelante" (Perkins, 2016: 156).

Ya desde el inicio, el caso clínico debe configurarse con los elementos del modelo disciplinar seleccionado para poder resolverlo y es deseable que incluya los diferentes tipos de competencias para luego poder hacer diferentes recortes en función del diseño de escenarios de simulación según corresponda al grado de avance de los/as estudiantes.

Segundo principio: Hacer que el juego valga la pena: Para que esto suceda son necesarias las versiones para principiantes en tanto "son la clave para lograr que el enfoque del aprendizaje pleno sea práctico y efectivo" (2016: 61) y se destaca el hecho de mantener un nivel razonable de desafío y prestar atención a lo que los/as estudiantes ya aprendieron.

Particularmente, en lo que hace al diseño de escenarios, Peña Jiménez y Amador Aguilar (2015: 156), indican que sean adecuados al nivel de complejidad que requieren los/as estudiantes en razón de sus conocimientos y experiencias previas tanto como de los objetivos de aprendizaje que se postulen.

A continuación, se brindan ejemplos de cómo a partir de un mismo caso clínico que se describe como anexo- se pueden derivar escenarios de simulación para los tres años de la Tecnicatura en Enfermería y con diferentes tipos de competencias:

- Primer año: Admisión de la paciente. Control signos vitales. Se pueden agregar/quitar datos. Ejemplo en relación al estado de higiene de la paciente (para planificar un escenario de baño en cama) o gravedad de su cuadro clínico (para no complejizar el caso)

- Segundo año: Administración de medicación. Colocación de vía IV. Habilidades de comunicación con los familiares. Habilidades de comunicación con otros profesionales

- $\quad$ Tercer año: el ejemplo de maniobras destinadas a valorar la salud maternofetal específicamente. También en relación a la Lactancia materna y la planificación familiar. 
Tercer principio: Trabajar las partes difíciles: En este caso, aparece otra de las etapas de la Simulación Clínica cuando se hace mención al Psicólogo cognitivo K. Anders Ericsson y al concepto de práctica deliberada en relación a lograr un mejor desempeño que "se verifica sólo gracias al esfuerzo consciente invertido en comprender cabalmente la tarea y reestructurar los antiguos desempeños" (Perkins, 2006: 108).

Según el recorte seleccionado para el diseño del escenario de Simulación puede ser necesario por ejemplo trabajar en relación al mantenimiento de la asepsia o medidas de bioseguridad o en relación a la anamnesis; es decir puede suceder que haya que recurrir a repasar aspectos de alguna técnica coadyuvante a la que se está realizando o identificar algún momento específico de la técnica en sí misma sobre la cual trabajar en forma más específica tanto como reforzar los elementos que constituyen el diagnóstico de Enfermería o el hecho de dirigirse a la persona por su nombre o apellido.

En lo que hace a trabajar las partes difíciles se ponen en juego las habilidades para el coaching y la retroalimentación ya que particularmente en esta última "la retroalimentación de correcto e incorrecto no proporciona suficiente información a los estudiantes" según Perkins (2006: 112).

Por una parte, no está de más recordar que el verbo to coach indica tanto tutela como adiestramiento así como dar indicaciones o comunicar hechos y el fin último es "liberar el potencial de una persona para alcanzar el máximo de su rendimiento" (Whitmore, 2011: 20), es por esto por lo que hay que ir más allá de un escueto: "está bien o está mal" como devolución a los/as estudiantes.

De la misma forma, en relación a la retroalimentación entendida como una información específica que permite clarificar una brecha entre un rendimiento estándar y el observado, se reconoce que "un estudiante debe recibir formación que desarrolle sus habilidades, conocimientos y aptitudes relacionadas a la profesión de estudio" (Ceccarelli Calle, 2014: 129).

Es decir, tanto el coaching como el feedback ponen el centro de atención en el/la estudiante, en que sean ellos/ellas los/las protagonistas de su propio proceso de aprendizaje, con lo cual el rol de los/las docentes tienden a ser de facilitador y por eso se acuerda con Perkins en que el binomio correcto/incorrecto no se adecua a estas circunstancias.

También cabe recordar que, así como la Simulación Clínica impacta en la enseñanza y el aprendizaje, resulta imposible separar a la etapa de evaluación que también tiene que ser revisada y alejada de los modelos tradicionales tendiendo a la autoevaluación lo cual deriva en la importancia del debriefing. El valor de trabajar las partes difíciles es tal que incluso la idea es preverlas e incorporarlas de forma planificada en el escenario previsto.

Cuarto principio: Jugar de visitante: Según Perkins, "La educación tiene como meta capacitar a los estudiantes con habilidades, conocimiento y comprensión para que los empleen en otros contextos" (2016: 145). En Enfermería ese otro momento y otro lugar 
alude, en primera instancia a la práctica profesionalizante y una vez egresado/a, a los ámbitos laborales.

Por lo pronto, al momento de realizar las prácticas profesionalizantes existen diferentes inconvenientes algunos más generales como la escasa posibilidad de cubrir las experiencias en cuanto al cuidado y otros más particulares como disposiciones de los hospitales docentes. Esta situación es mencionada por quienes afirman que "la entrada de estudiantes de pregrado a hospitales públicos y privados para valoración de pacientes se encuentra más restringida" (Bravo Zuñiga y col, 2018: 185).

En este jugar de visitante hay tiempo disponible para detener la actividad y propiciar espacios para la repetición y la retroalimentación; según Bland, Topping y Wood (2011), estas actividades pueden por un lado reducir el stress durante el proceso de enseñanza-aprendizaje-evaluación y por otro lado aumentar la confianza en los/as estudiante cuestiones que tenderían a confirmarse mediante estudios de investigación.

Quinto principio: Descubrir el juego oculto: Perkins (2016) describe cinco formas en las que este ítem se desarrolla: debajo de la alfombra de la simplicidad, lejos del sendero del sentido común, dentro de los márgenes de "con esto alcanza", bajo el manto de lo tácito y más allá del horizonte de la disposición. Todas ellas tienen su génesis en el contexto de la relación pedagógica.

Cabe destacar que en la etapa conocida como prebriefing o en la introducción a la actividad de simulación "se establecen las reglas del juego, se clarifican las limitaciones que tiene la simulación, se establece un ambiente constructivo y seguro en el que los alumnos puedan participar (...) se establecen los roles y responsabilidades (...)" (Fraga, 2018: 1).

Sin embargo, aún quedan cuestiones relacionadas a la forma en que la Simulación Clínica aparece en la currícula o relacionadas al plan de estudios en tanto las correlatividades que se plantean; por otra parte, también dan cuentan de este juego oculto, las planificaciones de cátedra y condiciones de cursado/recuperación de las diferentes actividades y por último pero no menos importante las estrategias de enseñanza en tanto "decisiones que toma el docente con el fin de promover el aprendizaje en los alumnos" (PRONAFE, 2018: 3).

Con relación a lo anterior, Urra Medina y colaboradores (2017) indican que, en este proceso de incorporar la Simulación Clínica en la currícula, un punto crítico es revisar la forma en la que se involucra al estudiante en el proceso de aprendizaje.

El rol de los/as estudiantes debe ser decididamente activo en pos de "reconocer que los planteamientos curriculares de la disciplina de Enfermería ya no pueden seguir encasillados en una concepción bancaria, memorística, pasiva y receptiva del sujeto que aprende" (Bernal Becerril y Ponce Gómez, 2009: 36); por lo tanto, esto además requiere de un acompañamiento institucional mediante toma de decisiones de tipo político y de gestión. 
En consonancia con lo anterior, se reconoce que "disponer de un programa de Enfermería, un espacio físico, simuladores y guías permite incluir herramientas pedagógicas como la simulación clínica. Sin embargo, se requiere asumir procesos liderados por personas interesadas en investigación y pedagogías innovadoras" (Niño Herrera y col, 2015: 975). Y en este punto la principal crítica va para instituciones que aún admiten esquemas rígidos y tradicionales para el proceso enseñanza-aprendizajeevaluación cosa que el Covid-19 vino a replantear con la necesidad de utilizar recursos tecnológicos e informáticos.

En el mismo sentido, Cardoso Delia y colaboradores (2017: 3) especifican diferentes objetivos muy claros en el diseño de políticas institucionales; entre ellos: incorporar la Simulación Clínica como estrategia pedagógica en el marco de un modelo educativo constructivista; formar competencias técnicas, cognitivas y actitudinales en los estudiantes y dos cuestiones que en pleno siglo XXI no resultan menores: producir material tecnológico audiovisual y establecer vínculos entre las organizaciones.

En síntesis: "la innovación de nuevas metodologías educativas (...) forja a que las instituciones educativas superiores emprendan sistemas de autorreflexión de sus estructuras pedagógicas y praxis docente que con urgencia requieren modelos educativos que generen estímulos motivacionales y el trabajo autónomo" (Bravo Zuñiga y colaboradores, 2018: 186)

Sexto principio: Aprender del equipo y de los otros equipos: Perkins (2016) enfatiza no sólo la construcción del conocimiento sino también la comprensión en tanto emprendimiento colectivo en contraposición al aprendizaje en solitario. Es en este contexto en que aparece el concepto de error como fuente de aprendizaje y la instancia del debriefing.

La Simulación Clínica introduce al error como un medio de aprendizaje lo que conlleva necesariamente a revisar desde una nueva óptica los procesos formativos conjuntamente con el énfasis en el autoaprendizaje (Urra Medina y col, 2017: 124). Se tiende a que los/as propios estudiantes se percaten del error y no a señalarlo desde el ejercicio de la docencia.

En cuanto a la instancia de debriefing, concebida como "conversación entre dos o más personas donde se analizan los detalles de un caso real o simulado para mejorar o sostener el rendimiento en situaciones similares en el futuro" (PRONAFE, 2019: 3) se destaca el componente reflexivo hacia la promoción de un cambio en la conducta de los/as participantes y la remisión a un marco de pensamiento.

Además, lo que se verifica es también la relación con el primer principio de Perkins (2006) ya que al mencionar que el conocimiento pone de relevancia el contexto y la cultura de la situación que genera la práctica simulada, esto conlleva a que el diseño de los escenarios a partir de un caso clínico sea familiar a docentes y estudiantes a partir del cual poder representarse el cuidado; que la situación hipotética sea lo más auténtica posible.

Sin embargo, lo anterior conlleva un riesgo que es advertido tanto por Perkins como por Peña Jiménez y Amador Aguilar ya que el primero afirma que "el aprendizaje 
social puede también reforzar la mediocridad (...)" (2006: 211) y los segundos autores señalan a la enseñanza situada como un "proceso de enculturación en el cual los estudiantes se integran de forma gradual a una comunidad o cultura de prácticas sociales" (2015: 155). Es decir, corresponderá a los/as docentes esta cuestión de remarcar el ejercicio profesional responsable a través del armado de escenarios y la planificación de las diferentes fases de la Simulación Clínica.

Séptimo principio: Aprender el juego del aprendizaje: Perkins (2006) ejemplifica este punto refiriéndose al "efecto acompañante" y señalando que en algunos contextos de aprendizaje se les niega a los estudiantes el rol de conductores según la cultura del entorno. Esto es fácilmente apreciable en las siguientes imágenes donde en las dos primeras imágenes (de izquierda a derecha) los/as estudiantes aparecen en un rol pasivo más allá de incluirse simuladores en la práctica de laboratorio y en las otras dos se muestran en actitud de resolver una consigna correspondiente a un caso dado.

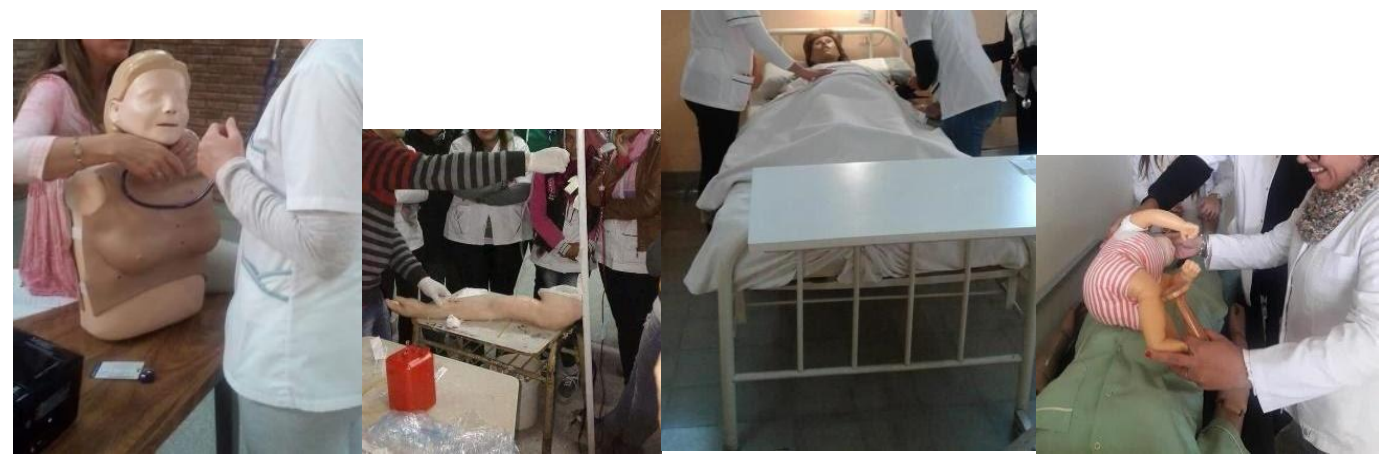

De izquierda a derecha: Taller de auscultación de ruidos cardíacos y pulmonares, Simulación de colocación de vía endovenosa, Taller de cuidados en Gestosis y Taller de control de salud materno-fetal. En las dos imágenes de la izquierda se observa, en contraste con las otras dos, el efecto acompañante al que alude Perkins.

En este punto se destaca la posibilidad de relación entre diversos espacios formativos: Anatomía y fisiología, Bioética y Deontología, Alimentación, Nutrición y dietética, Enfermería Comunitaria, Salud Pública, Farmacología, Aspectos psicosociales y culturales del desarrollo, Conocimiento de la realidad en el contexto global, Cuidados basados en la evidencia, Proceso social aplicado al contexto de salud. De hecho, el propio Perkins (2006) alude a un juego integrador, donde confluyen varias disciplinas. Es decir, en el diseño de escenarios fácilmente se pueden incorporar a modo de refuerzo saberes previos de diversos espacios formativos e incluso pueden participar de la experiencia docentes de distintas asignaturas que pueden aprovechar el espacio para desarrollar o acreditar o evaluar algún contenido.

Por ejemplo: el hecho de enseñar el lavado de manos se puede realizar a partir de relatar el caso de una persona que ha recibido un trasplante de riñón y el riesgo de adquirir una infección intrahospitalaria relacionada con la disminución de su sistema inmune. En ese caso se pueden enlazar contenidos de Microbiología (cuál es el microorganismo más factible de contaminar); con Ética y Deontología (el principio de no hacer daño); con Farmacología (en relación a la administración de medicación para evitar el rechazo del órgano); con Proceso Social aplicado al contexto de salud (por los diferentes 
roles en el entorno hospitalario). Es decir, varios espacios formativos pueden compartir el mismo caso y eso generaría una enorme posibilidad de vincularse entre los/as responsables de los mismos para acordar actividades en conjunto.

Desde este trabajo se concibe que la Simulación Clínica brinda la oportunidad de diálogo entre los/as docentes ya sea del mismo año o de años diferentes e incluso se podrían planificar escenarios en los que se compartan espacios ya sea de la propia disciplina como de diferentes disciplinas. Un ejemplo lo brinda el artículo de David Stonehouse donde detalla el Modelo de Roper, Logan y Tierney para trabajadores de apoyo en el ámbito de la salud: "los -trabajadores de apoyo trabajan en estrecha colaboración con colegas de Enfermería que implementan el proceso de Enfermería para brindar atención de calidad" (2017: 1). Si una institución cuenta con carreras de Medicina, Partería y Enfermería pueden planificar la recepción del recién nacido en conjunto.

\section{En primera persona: La palabra de un Coordinador de un Centro de Simulación}

El Licenciado en Enfermería Emiliano Arguello es docente desde hace 8 años, durante los cuales ha utilizado la Simulación Clínica. Durante 3 años estuvo a cargo del Centro de Simulación de la Universidad Adventista del Plata en la localidad de Villa Libertador General San Martín en la provincia de Entre Ríos, República Argentina. En el presente artículo relata su experiencia a la luz de la incorporación del enfoque pedagógico de David Perkins.

Estar a cargo de la coordinación de un centro de simulación implica ser responsable de una gran cantidad de actividades, muchas de las cuales son lo que se podría denominar "operativas", tales como garantizar que haya suficientes materiales para la recreación de escenarios, que los equipos estén funcionando adecuadamente, así como prestar colaboración a los docentes para que el escenario pensado por ellos tenga todas las herramientas y el contexto adecuado para alcanzar los objetivos planteados.

Otra importante responsabilidad del coordinador es motivar a cada docente para que los contenidos teóricos y prácticos puedan ser adaptados a fin de ser abordados por medio de la simulación. Actualmente aún existe gran cantidad de docentes que opta por la exposición teórica de los contenidos, por lo cual una gran actividad es poder favorecer que cada uno de los docentes pueda modificar su estructura mental para ver su materia de manera diferente e implementar así distintas estrategias pedagógicas como la simulación clínica.

Mucho de lo que se relaciona con la dinámica de gestión de un centro de simulación depende en gran parte directamente de la motivación de los docentes para que utilicen esta estrategia educativa en sus clases, favorecer la creación de escenarios requiere experiencia clínica para poder hacerlo ya que implica recurrir a la recreación en sus mentes de experiencias previas y favorece el plasmar los conocimientos de cada materia en las actuaciones de la vida real. 
Hay una gran cantidad de actividades docentes que se deben realizar en los centros de simulación. En principio, el docente debe comprender que a pesar que el escenario de simulación recrea una actividad en la cual requiere distintas competencias, el objetivo que se plantea para esa clase debe estar inducido y orientado. El docente debe simplificar al máximo lo que quieren lograr como objetivo dentro del escenario y colocarlo como punto de valoración en ese momento; esto no significa que no tienen que corregir si hay errores en el escenario, sino que el objetivo del mismo es el aprendizaje por competencias. En este sentido necesitamos como docentes realizar mucha más investigación de cuál es el impacto que tiene el desarrollo de simulación en el aprendizaje y más que nada en la capacidad que generamos en el alumno a auto reflexionar, modificar sus conductas y técnicas.

Cuando los docentes logran internalizar y utilizar las simulaciones de forma sistemática, el enfoque metodológico resulta de gran valor para nuclear a través de toda una carrera que los docentes utilicen las mismas formas y métodos para lograr el aprendizaje de competencias, habilidades y pensamiento crítico. -Entonces aplicando metodología educativa podemos lograr mejores planificaciones mejores simulaciones y aumentar el logro de objetivos y la satisfacción de los/as estudiantes.

La adopción enfoque metodológico de Perkins nos ayudaría muchísimo como profesionales de la salud en el ejercicio de la docencia, a poder diagramar de qué forma y cuáles serían los pasos a seguir para mejorar el aprendizaje en simulación de las competencias necesarias para ser profesionales. Cada uno de los pasos puedes ayudar muchísimo a establecer un aspecto tanto del aprendizaje de la técnica como del pensamiento crítico pero así también desarrollando una forma de aprendizaje que constantemente nos lleve a la auto reflexión y nos ayude a visualizar la posibilidad de aprender en cualquier situación.

Realmente los pasos que propone Perkins para el aprendizaje se ven muy bien reflejados en la manera de aprender en simulación; podría decirse que los primeros tres pasos -que se refieren al jugar el juegos completo, que el juegos valga la pena y trabajar las partes difíciles- suelen ser lo que más realizamos en simulación en las carreras de pregrado o por lo menos lo que deberíamos hacer ya que en muchos lugares no se realiza de esta forma y solamente se ven habilidades y se trata de tener experticia en estas habilidades que se limitan a lo procedimental. De una manera completa y entre medio incorporar otras habilidades nos ayuda a que el aprendizaje sea más integral y completo.

El paso que propone Perkins de jugar de visitante nos da la gran idea de buscar la manera de hacer transferencia con esto que aprendemos en el juego completo; la transferencia es muy importante para poder afianzar ese conocimiento y buscar la manera de realizarlo en escenarios similares donde suceden las prácticas reales; esto puede acercarnos nuestro objetivo. Al comienzo, nos sentimos inseguros en el entorno, pero sí ya hemos realizado este escenario, luego nos adaptamos a ese lugar y podemos jugar con 
diferentes compañeros y en diferentes situaciones para lograr un aprendizaje significativo y cíclico.

Los siguientes tres pasos -descubrir el juegos oculto, aprender del equipo y de los otros equipos y aprender el juego del aprendizaje- son pasos que entiendo que van más relacionados con el aprendizaje de habilidades blandas -que de todas maneras pueden incluirse incluso en escenarios diseñados para principiantes- cómo son el pensamiento crítico el trabajo colaborativo las relaciones sociales el trabajo en equipo y al final de todo esto aprender el respeto hacia el equipo de trabajo, la autorreflexión y la forma fantástica en la que el aprendizaje se realiza.

Los estudiantes, en tanto participantes de las simulaciones, aprecian muchísimo el aprendizaje en estos formatos. Los alumnos de pregrado han demostrado de diferentes maneras la valoración por la seguridad que les brinda esta estrategia educativa, ya que sienten haberlas realizado como en los nosocomios con el agregado de permitirse el error y favorecer la seguridad de las personas. Los alumnos de posgrado han referido también el gran aprendizaje que se obtiene al realizar cuidados conocidos en ambientes o escenarios desconocidos con colegas desconocidos y cómo esto impacta en la afinación de las habilidades relacionales y de equipo, a la misma vez que se agregan conocimientos específicos del curso. También he podido presenciar el impacto de grupos en cuyas instituciones no aprenden con esta estrategia por diferentes motivos, y que, al ponerlos en los escenarios no saben adecuadamente reaccionar aunque hayan visto el contenido teórico.

La utilización de la simulación ha colaborado muchísimo a romper la estructura disciplinar y comenzar a apreciar el trabajo multidisciplinar y cooperativo; desde los directivos, hasta docentes y alumnos, pueden observar el gran avance que se logra cuando determinamos el equipo por encima de la individualidad en la profesión.

La innovación educativa como el resultado del maridaje entre las funciones de investigación y docencia

Como ya se señaló anteriormente, la preocupación para la continuidad de la investigación pasaba por revisar la forma de incorporar la Simulación Clínica al currículum del pregrado en Enfermería. Se consideró que "prácticas con muñecos" y la enumeración de técnicas y procedimientos como unidades de las planificaciones de cátedra reforzaba el modelo biomédico y en la práctica en el aula se mantenían los roles tradicionales de docentes y estudiantes.

Se acuerda en que "es necesario intensificar el diálogo entre ambos campos del saber de manera tal que los aportes de la didáctica constructivista puedan ser considerados en términos de su impacto sobre la calidad de los aprendizajes (...)" (Ferrero, 2018: 56) y por lo tanto se insiste en que la Simulación Clínica requiere de enfoque pedagógico, modelo disciplinar e investigación en forma paralela a su implementación para contribuir a su especificidad en las ciencias de la salud. 
Es por esto que se entiende como apropiado el enfoque pedagógico del Aprendizaje Pleno que, por una parte, al proponer jugar el juego completo habilita la incorporación de un modelo disciplinar que resalte la adquisición de competencias cognitivas y no sólo procedimentales en el acto de cuidar. Por otra parte, en cada uno de los siete principios enumerados por Perkins (2016) se manifiestan las diferentes instancias de la estrategia educativa de la Simulación Clínica.

\section{Referencias bibliográficas}

BÁEZ-HERNÁNDEZ, F., NAVA-NAVARRO, V., RAMOS-CEDEÑO, L. y MEDINA-LÓPEZ, O. (2009) "El significado de cuidado en la práctica profesional de Enfermería". En Chía, Año 9, Vol 9 (2): 127-134 disponible en www.scielo.org.co. Los autores citan un artículo de Medina J del año 1999.

BERNAL BECERRIL, M. y PONCE GÓMEZ, G. (2009) “Propuesta para la enseñanza del cuidado enfermero" En Revista de Enfermería Universitaria. enero-marzo, Vol 6, año 6 (1): 33 a 41. Disponible en www.redalyc.org

BLAND, A., TOPPING, A. y WOOD, B. (2011) "A concept analysis of simulation as a learning strategy in the education of undergraduate nursing students". En Nurse Education Today. UK. Elsevier 31: 664-670 disponible en www.researchgate.net

BRAVO ZUÑIGA, B., GONZÁLEZ PEÑAFIEL, A. y VALLE FLORES, J. (2018). "Ambientes y diseño de escenarios en el aprendizaje basado en la simulación". En Revista Pedagógica de la Universidad de Cienfuegos, oct-enero, Vol 14 (61): 184-90. Disponible en www.scielo.sld.cu

CARDOZO, D., LAPINSKI, S., NUÑEZ, C., SILVA, L., BLANCO, C. y YESA, J. (2017). “Cambios en la metodología de enseñanza aprendizaje: aplicación de la Simulación". Ponencia presentada en el XIV Encuentro de Universidades Nacionales. 08 al 10 noviembre en Posadas, Misiones, Argentina: 1-8 disponible en www.fhycs.edu.ar

CECCARELLI CALLE, J. (2014). “Feedback en educación clínica”. En Revista Estomatología Herediana. abril-jun; 24 (2): 127-132. Disponible en www.redalyc.org

COBO ROMANÍ, C. y MORAVEC, J. (2011). Aprendizaje invisible. Hacia una nueva ecología de la educación. Col•lecció Transmedia XXI. Laboratori de Mitjans Interactius /Publicacions i Edicions de la Universitat de Barcelona: Barcelona.

COLL, C. (1997). Aprendizaje escolar y construcción del conocimiento. México: Paidós.

DARÓS, W. (1994). Fundamentos antropológico - sociales de la educación. Libertador San Martín: Universidad Adventista del Plata.

DELORS, J. (1997). La educación encierra un tesoro. México: Correo de la UNESCO.

DUART, J. y SANGRÁ, A. (comp.) (2005). Aprender en la virtualidad. Barcelona: Gedisa. 
FERREIRO GRAVIE, R. (2007). "Una visión de conjunto a una de las alternativas educativas más impactante de los últimos años: El aprendizaje cooperativo" en Revista Electrónica de Investigación Educativa, 9(2) disponible en www.redalyc.org

FERRERO F. (2018). ¿Puede la Simulación Clínica contribuir al aprendizaje significativo de competencias educativas? Una aproximación constructivista. Disponible en www.medigraphic.com

FRAGA, J. M. (2018). "Sesiones con simulación. Aspectos Generales". Material del Curso virtual "Introducción a la Simulación Clínica”. Udemy.

FERREIRO, R. (2007). Aprendizaje cooperativo. Revista Electrónica de Investigación Educativa, 9 (2). Disponible en: http://redie.uabc.mx/vol9no2/contenidoferreiro.html

FIRPO, R. (2018): Proyecto de investigación "La utilización de simuladores en la enseñanza en Enfermería: salir de la racionalidad instrumental a través de nuevas prácticas pedagógicas". Instituto Superior Diamante. Disposición 037/17 Dirección de Educación Superior. Consejo General de Educación. Entre Ríos.

INACSL Standards Committee (2016): INACSL Standards of best practice Simulation. Simulation Glossary. En Clinical Simulation in Nursing 12 (S), S 39-47 disponible en http://dx.doi.org

INSTITUTO NACIONAL DE EDUCACIÓN TÉCNICA. Programa Nacional de Formación de Enfermeros/as, "Curso de didáctica y práctica docente en Enfermería". 2018, módulo 2, unidad 3, p 3 cita a Anijovich y Mora, Estrategias de enseñanza: otra mirada del quehacer en el aula 2010: 9

INSTITUTO NACIONAL DE EDUCACIÓN TÉCNICA. Programa Nacional de formación de Enfermería. Curso Simulación en Acción, año 2019. Evaluación del debriefing para la Simulación en Salud (EDSS) Manual del Evaluador. Center for Medical Simulation. Hospital virtual de Valdecilla, p 2

MARCO STIEFEL, B. (2008). Competencias básicas: Hacia un nuevo paradigma educativo. Madrid: Narcea.

MEDINA GONZÁLEZ, S. (2017). Aprendizaje colaborativo. Educación, (23), 101-105. https://doi.org/10.33539/educacion.2017.n23.1175

NIÑO HERRERA, C., VARGAS MOLINA, N. y BARRAGÁN BECERRA, J. (2015) "Fortalecimiento de la Simulación Clínica como herramienta pedagógica en Enfermería: experiencia de internado". En Revista Cuidarte, vol. 6 (1): 970-975, Universidad de Santander, Colombia. Disponible en www.redalyc.org

PERKINS, D. (2016). El Aprendizaje Pleno. Principios de la enseñanza para transformar la educación. 1ra edición en español. Argentina: Ed. Paidós.

PERRENOUD, P. (2007). Diez nuevas competencias para enseñar. Barcelona: Grao.

PIÑA JIMÉNEZ, I. y AMADOR AGUILAR, R. (2015). “La enseñanza de la Enfermería con simuladores. Consideraciones teórico-pedagógicas para perfilar un modelo didáctico". En Enfermería Universitaria. México. julio-sept; 12 (3): 152-159 disponible en www.revista-enfermeria.unam.mx 
REVELO-SÁNCHEZ, O., COLLAZOS ORDÓÑEZ, C. y JIMÉNEZ TOLEDO, J. (2018). “EI trabajo colaborativo como estrategia didáctica para la enseñanza/aprendizaje de la programación: una revisión sistemática de literatura" en revista Tecnológicas 21(41), 115-134 disponible en www.scielo.org.co.

ROPER, N., LOGAN, W. y TIERNEY, A. (1993). Modelo de Enfermería. 3ra edición. México: Interamericana-McGraw-Hill.

SHARMA, R. (2017). "Emerging Innovative Teaching Strategies in Nursing" en JOJ Nursing and Health Care. april 21; 1 (2), p 01-03. Disponible en www.researchgate.net

SMITH, R. (2004). El proceso pedagógico ¿agonía o resurgimiento?: Una búsqueda desde la cosmovisión bíblica. Montemorelos, N. L., México: Universidad de Montemorelos.

STONEHOUSE, D. (2017). “Roper, Logan \& Tierney's Model of living and Model of nursing: A support workers guide", disponible en http://usir.salford.ac.uk

TRISCA, J. (2014). Competencias y grupos cooperativos. Montemorelos, México: Editorial Montemorelos.

URRA MEDINA, E., SANDOVAL BARRIENTOS, S., IRRIBARREN NAVARRO, F. (2017). "EI desafío y futuro de la simulación como estrategia de enseñanza en Enfermería" En Investigación en Educación Médica. abril-junio, vol. 6 (22): 119-125, Universidad Nacional Autónoma de México. Disponible en www.redalyc.org

WHITMORE, J. (2011). “¿Qué es el coaching?” Cap. 1 en Coaching. El método para mejorar el rendimiento de las personas. México: Editorial Paidós.

WOOLFOLK, A. (2010). Psicología Educativa. México: Prentice Hall.

ZABALA, A. y ARNAU, L. (2010). Cómo aprender y enseñar competencias. Barcelona: Graó.

\section{Anexo: Diseño de un Caso clínico para "jugar el juego completo"}

El caso que se detalla a continuación está realizado desde el Modelo de Vida de Roper, Logan y Tierney por lo que se presentan datos correspondientes a los diferentes componentes del mismo: etapa de la vida, actividades vitales, factores que influyen, nivel dependencia o independencia e individualidad de la vida. Se elige de forma deliberada un caso donde se trabaja con un evento fisiológico como es un embarazo y que por lo tanto puede ser útil para el diseño de escenarios de diferente complejidad.

Clara, 23 años, cursa semana 31 de G1 P0 A0. Vive en el Barrio Belgrano de la ciudad de Diamante en una casa prefabricada con su pareja de 28 años en el mismo terreno que habitan sus padres (madre de 45 años, Ama de casa y padre de 48 años, empleado en un comercio)

Su peso actual es de $72.600 \mathrm{~kg}$ (peso anterior al embarazo de $62 \mathrm{~kg}$ ) y mide 1.60 metros. Tiene realizadas 3 consultas prenatales en el Centro de salud; la última fue hace dos meses. FUM: refiere no recordar exactamente. Tiene secundario incompleto, es Ama de casa, está en pareja con el padre del niño/a desde hace 2 años. Refiere que fue un 
embarazo no planificado. No cuenta con Obra Social. En el día de la fecha comenzó a tener dificultad para respirar, dolor abdominal y presenta edema en miembros inferiores (leve, grado 2). Se la ingresa en la sala de maternidad del Hospital San José y está a la espera de ser trasladada a la ciudad de Paraná para ser atendida en el Hospital Materno-infantil San Roque. Sus parámetros vitales son: tensión arterial 150/85 mmHg; Frecuencia cardíaca: 85 latidos por minuto; Frecuencia respiratoria 20 respiraciones por minuto; Temperatura: 36.9 grados. No refiere pérdidas sanguíneas. Está acompañada por su madre (que interviene en todo momento durante la admisión de su hija, la toma de la mano y le acaricia la cabeza, le recuerda que "yo te dije que fueras al control" y "te vi los pies hinchados hace ya varios días, deberías haber descansado más") ya que su pareja está en un viaje por su ocupación laboral (camionero). No asistió aún al curso de preparto y no decidió sobre la lactancia

Refiere preocupación por el pronóstico del embarazo, pregunta si "va a llegar a término" y si esto que le sucede es porque se colocó la vacuna antigripal la semana pasada.

Las indicaciones son: Control de SV cada dos horas. Metildopa $250 \mathrm{mg} 1$ comprimido vía oral cada 12 horas. Dieta hiposódica y colocación de vía parenteral. En reposo hasta el momento de la derivación

* Jorge Omar Trisca: Doctor en Educación. Especialidad en Instrucción y Currículum. Docente-investigador. Autor de: Competencias y grupos cooperativos (2014), Estrategias y actividades para desarrollar competencias (2020, en edición). Actualmente investigador sobre Estrategias de enseñanza y aprendizaje, uso de la tecnología educativa en ambientes de aprendizaje y desarrollo de estrategias metacognitivas. [E-mail: trisca@um.edu.mx]

** Rosana Elisabet Firpo: Licenciada en Enfermería (Universidad de Buenos Aires). Técnica Universitaria en Comunicación Social (Unidad Académica Río Gallegos - Universidad Nacional de la Patagonia Austral). Docente-investigadora. Co-autora de: Pensar el cuidado (2008), Adolescentes y Autocuidado (2011) -el primer libro basado en un proyecto de Extensión de la UARG-UNPA-, El arte de cuidar (2012) y La cultura del cuidado del patrimonio histórico y la salud (2016). Autora del proyecto de investigación "La utilización de simuladores en la enseñanza en Enfermería: salir de la racionalidad instrumental a través de nuevas prácticas pedagógicas" (2018) desde el que se orientó la adopción del enfoque pedagógico de David Perkins para la Simulación Clínica en Enfermería. En Instagram: @cuidar.comunicación.[E-mail: rosanaelisabet@gmail.com] 
*** Emiliano Argüello: Licenciado en Enfermería (Universidad Nacional de Rosario). Especialista en cuidados críticos de Enfermería (Universidad Favaloro). Docente. Actual Coordinador de Enfermería de la Universidad de Montemorelos, Ex Director del Centro Interdisciplinario de Simulación en Salud en la Universidad Adventista del Plata en Villa Libertador General San Martín (ER). Coautor de: "Aprendizaje por competencias. La simulación de alta fidelidad en el ámbito de los cuidados críticos" Capítulo del Programa de Actualización de Enfermería en Cuidados Críticos de Editorial Panamericana. [E-mail: emilianoarguello@um.edu] 